\section{System for Cleavable Fc Fusion Proteins Using Tobacco Etch Virus (TEV) Protease}

BioTechniques 30:60-66 (January 2001)

\begin{abstract}
We describe a novel Fc fusion protein system that can be cleaved by tobacco etch virus (TEV) protease. This system is desirable because it takes advantage of the high specificity of TEV protease and its activity at $4^{\circ} \mathrm{C}$. We produced two TEV-Fc fusion proteins that contain the first three Ig domains and all six Ig domains of the cell adhesion molecule L1. Both proteins were efficiently cleaved by TEV protease at $4^{\circ} \mathrm{C}$. Functional analysis of the cleavage products in neurite outgrowth assays showed they had similar activities to their parental Fc fusion proteins. Therefore, TEV-Fc fusion proteins may increase the utility and flexibility of the Fc fusion protein system.
\end{abstract}

\section{INTRODUCTION}

Fc fusion proteins are a popular means of producing eukaryotic receptors and extracellular molecules (2). They are most commonly structured like IgG antibodies, in which the variable regions are replaced by a protein sequence of interest. The Fc region of these molecules serves as a tag for affinity purification and immunostaining and also confers a bivalent structure.

While the Fc region confers several useful properties to these fusion proteins, its presence can sometimes be problematic. In vivo, the bioavailability of Fc fusion proteins can theoretically be lowered by the Fc region, through binding of the proteins to cell surface Fc receptors. The branched, bivalent structure imposed by the Fc region complicates the use of Fc fusion proteins for structural analysis via electron microscopy or X-ray crystallography. Furthermore, the Fc region can sometimes reduce biological activity (3).

We were concerned with this possibility recently while conducting a structure-function analysis of the cell adhesion molecule L1 (4). The extracellular region of $\mathrm{L} 1$ can mediate several biological processes in the vertebrate nervous system, such as neurite outgrowth. We found that an Fc fusion protein containing the six Ig domains of L1 possessed neurite outgrowth activity at levels comparable to the native molecule, while fusion proteins containing fewer Ig domains were less active (4). While these results suggested that the six Ig domains of L1 were a functional unit, we could not exclude the possibility that the Fc region exerted an artifactual, negative influence on the shorter Fc fusion proteins, leading to a false-negative result.

Therefore, it was of value to analyze L1 fragments contained in our Fc fusion proteins without the presence of the Fc. Rather than resort to a different means of protein production, we designed a cleavable $\mathrm{Fc}$ fusion protein system in which the Fc region can be removed by tobacco etch virus (TEV) protease $(8,9)$. TEV protease is a com mercially available enzyme that is advantageous because of its high specificity for a seven amino acid recognition sequence and its activity at $4^{\circ} \mathrm{C}$ (9). It has been used successfully to remove other types of fusion tags, such as glutathione-S-transferase (GST) and poly-histidine $(7,8,10)$. To verify our earlier findings, we made two TEV-Fc fusion proteins containing the six $\mathrm{Ig}$ domains of human L1 (that was previously found to be bioactive) and the first three Ig domains (that was found to be inactive) (4). Cleavage of these TEV-Fc fusion proteins with TEV protease was then done to obtain L1 fragments in absence of the Fc. Finally, these fragments were assayed for neurite outgrowth activity, and the results confirmed our previous analysis of L1. These results demonstrated the usefulness of TEV-Fc fusion proteins for this and other applications.

\section{MATERIALS AND METHODS}

\section{Construction of TEV-Fc Plasmids}

The plasmid pCR/TEV-Fc was derived from the expression construct $\mathrm{pCR} / \mathrm{Fc}$ (4), which contains a human IgG cDNA cloned into pCR3.1 (Invit- rogen, Carlsbad, CA, USA). A doublestranded oligonucleotide encoding the TEV protease cleavage site was made by annealing the oligonucleotides $5^{\prime}$ GATCCCGAGAACCTGTACTTCCA GGGTACCGAA-3' (TEV_Sense) and 5'-GATCTTCGGTACCCTGGAAGTACAGGTTCTCGG-3' (TEV_Antisense). This was phosphorylated with polynucleotide kinase and ligated into pCR/Fc cut with $B a m H I$. Clones containing the insert were identified by $K p n \mathrm{I} / S t u \mathrm{I}$ double digest, and the orientation was confirmed by sequencing. To construct L1-TEV-Fc expressing plasmids, PCR products encoding the first three (L1-13) or all six Ig domains (L116) of human L1 were produced with $P f u$ DNA polymerase (Stratagene, La Jolla, CA, USA) as described previously (4). The PCR products were digested with $E c o$ RI and $B c l I$ and ligated into pCR/TEV-Fc that was cut with EcoRI and BamHI. Finally, positive clones were identified by HindIII digests. The resultant plasmids were called $\mathrm{pCR} / \mathrm{L} 1$ 13TEV-Fc and pCR/L1-16TEV-Fc.

\section{Protein Production}

HEK 293 cells were transfected with pCR/L1-13TEV-Fc or pCR/L1$16 \mathrm{TEV}-\mathrm{Fc}$, and stable clones were isolated by selection with $1 \mathrm{mg} / \mathrm{mL}$ effective G418 (Life Technologies, Rockville, MD, USA). Cell lines that highly express L1-13TEV-Fc or L1-16TEV-Fc were identified by ELISA. These were passaged into $15-\mathrm{cm}$ tissue culture plates (Falcon, Lincoln Park, NJ, USA) and cultured in DMEM, 10\% IgG-depleted fetal calm serum (FCS) (Life Technologies), 2 mM 1-Gln, $55 \mu \mathrm{g} / \mathrm{mL}$ gentamicin. Note that IgG-depleted FCS was used to avoid contamination of Fc fusion protein preparations with bovine Igs (that are abundant in standard FCS). At one-week intervals, the media was collected and the cells were refed; such terminal cultures typically lasted for four weeks. Cultured supernatants containing $\mathrm{Fc}$ fusion protein were spun at $4500 \times g$ for $15 \mathrm{~min}$ and decanted to remove cellular debris. The protein in the supernatant was then precipitated by addition of $390 \mathrm{~g} / \mathrm{L}$ ammonium sulfate (Sigma, St. Louis, MO, USA). The precipitate was collected by centrifugation at $10000 \times \mathrm{g}$ for $15 \mathrm{~min}$ 
at $4{ }^{\circ} \mathrm{C}$, resuspended in PBS $(40 \mathrm{~mL} / \mathrm{L}$ cultured supernatant) and then dialyzed against 50 volumes PBS with three changes. Finally, the amount of Fc fusion protein $/ \mathrm{mL}$ ammonium sulfate dialysate was determined by immunoprecipitation of $1 \mathrm{~mL}$ of the dialysate with $30 \mu \mathrm{L}$ protein-A Sepharose ${ }^{\circledR}$ slurry (Pierce Chemical, Rockford, IL, USA). To purify L1-13TEV-Fc and L116TEV-Fc, ammonium sulfate dialysates were incubated with protein-A Sepharose, and the proteins were eluted with $100 \mathrm{mM}$ glycine, $\mathrm{pH} 2.8$, as described for other $\mathrm{Fc}$ fusion proteins (11). Typical yields were $1-3 \mathrm{mg} / \mathrm{L}$ cultured supernatant.

\section{Cleavage of TEV-Fc Fusion Proteins}

After ammonium sulfate precipitation and dialysis, preparations of L113TEV-Fc or L1-16TEV-Fc were incu- bated with protein-A Sepharose beads (150 $\mu \mathrm{L}$ packed beads $/ 50 \mathrm{~mL}$ dialysate) in a $50-\mathrm{mL}$ conical tube (Falcon) at $4^{\circ} \mathrm{C}$ overnight. The beads were then washed twice with $10 \mathrm{~mL} 6 \times \mathrm{PBS}, 0.1 \%$ Triton ${ }^{\circledR} \mathrm{X}-100$, twice with $10 \mathrm{~mL} 1 \times$ PBS, $0.5 \%$ Triton $X-100$, and four times with $10 \mathrm{~mL}$ PBS. The beads were resuspended in $0.5-1 \mathrm{~mL} \mathrm{PBS}$ and transferred to a 1.5-mL centrifuge tube. TEV protease (Life Technologies) was then added, and the slurry was incubated with rocking at $4^{\circ} \mathrm{C}$ for $6-21 \mathrm{~h}$. It was determined that $0.25 \mathrm{U}$ protease/ $\mu \mathrm{g}$ TEV-Fc fusion protein is optimal for efficient cleavage under these conditions (data not shown). Equal aliquots of slurry were collected and analyzed on SDS-PAGE gels stained with SYPRO ${ }^{\circledR}$ Orange (Molecular Probes, Eugene, OR, USA) to measure cleavage efficiency. The cleavage reaction supernatant, containing the cleavage product, was separated from the beads by passing the slurry through a disposable plastic column (Bio-Rad Laboratories, Hercules, CA, USA). The protein concentration was measured using Bradford assay (Bio-Rad Laboratories), and the purity was assessed by SDSPAGE gels stained with SYPRO Orange. Finally, the proteins were analyzed via western blot with anti-human Fc antibody (Jackson ImmunoResearch, West Grove, PA, USA) or with Neuro 4.1, a monoclonal antibody specific for human L1 (12).

\section{Removal of TEV Protease}

To remove TEV protease, protein preparations were incubated with 10-20 $\mu \mathrm{L}$ TALON ${ }^{\circledR}$ metal affinity resin (Clontech Laboratories, Palo Alto, CA, USA) for $10 \mathrm{~min}$ at room temperature under agitation. The beads were re-
A

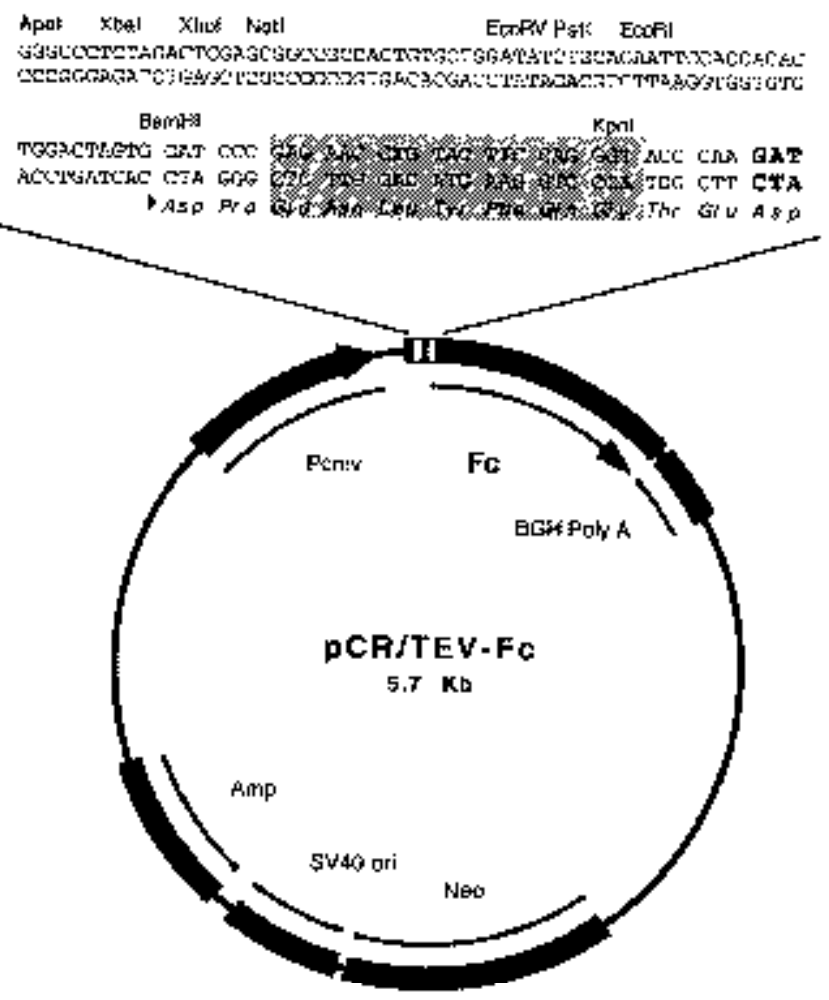

B
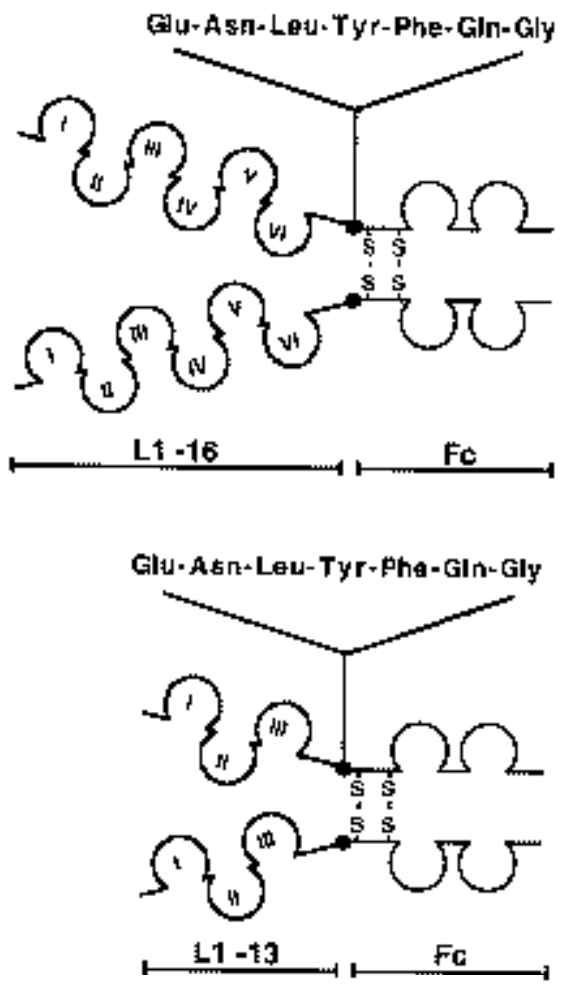

Figure 1. Models of plasmid and proteins. (A) Plasmid map of pCR/TEV-Fc. The multiple cloning site (striped box) is detailed above the plasmid. Codons encoding the TEV protease cleavage site are highlighted in gray, and the first codon of the human Fc region is shown in boldface. (B) Structure of L1-16TEV-Fc and L1-13TEV-Fc. The proteins contain all six (L1-16) or the first three (L1-13) Ig domains of human L1, which are indicated by numbered loops. The TEV protease cleavage site is represented by a filled circle that is located $\mathrm{N}$ terminal to the $\mathrm{Fc}$ region and is detailed above each drawing. 
moved by centrifugation, and the supernatant, containing the cleavage product, was collected. To verify removal of the protease, equivalent aliquots, obtained before and after addition of the metal affinity resin, were analyzed via SDS-PAGE.

\section{Reaggregate Neurite Outgrowth Assay}

Reaggregate neurite outgrowth was done essentially as described (4). Briefly, P0-P4 mouse cerebellar reaggregates were plated on polystyrene dishes (Falcon) coated with $10 \mu \mathrm{g} / \mathrm{mL}$ poly-1-lysine (Sigma) and 0-600 nM L1-TEV-Fcs or L1 cleavage products. After $16 \mathrm{~h}$ incubation, the length of the tenth longest neurite of each reaggregate was measured using NIH Image software as described (4).

\section{RESULTS AND DISCUSSION}

To produce TEV-Fc fusion proteins, the expression construct pCR/TEV-Fc was made (Figure 1A). This plasmid encodes the $\mathrm{Fc}$ region of human $\mathrm{IgG}$ fused to a sequence containing the TEV protease site. Using this plasmid, two TEV-Fc fusion proteins were generated: L1-16TEV-Fc, which contains all six Ig domains of human L1, and L113TEV-Fc, which contains the first three Ig domains (Figure 1B). The TEV protease site resides in the "hinge" region of the proteins, between the L1 and Fc sequences. L1-16TEV-Fc and L1-13TEV-Fc migrated at approximately 140 and $90 \mathrm{kDa}$, respectively, and were recognized by anti-human L1 and anti-human Fc antibodies (Figure 2 and data not shown).

A purification and cleavage method for L1-16TEV-Fc and L1-13TEV-Fc was devised. The proteins were absorbed to protein-A Sepharose beads, and the beads were washed with PBS to remove unbound contaminants. TEV protease was then added to the slurry, and the reaction was incubated at $4^{\circ} \mathrm{C}$ under agitation. After $6 \mathrm{~h}$, a significant amount of cleavage was observed when analyzed by SDS-PAGE (Figure 2). L116TEV-Fc was cleaved into two bands of approximately 110 and $30 \mathrm{kDa}$ that were identified by western blot as the
L1-16 fragment and the Fc region, respectively (Figure 2, A and B). The Fc region remained bound to the protein- $A$ beads, while the L1-16 fragment was liberated into the reaction supernatant (Figure 2B). A minor fraction of L116TEV-Fc (about 10\%) appeared to be resistant to cleavage. However, minimizing the amount of protein-A beads used to absorb the Fc fusion protein could reduce this population (data not shown). This observation suggests that some protein binding sites on or within the protein-A beads are inaccessible to the protease.

L1-13TEV-Fc was also efficiently cleaved by TEV protease. The intact fusion protein, which migrated at about $90 \mathrm{kDa}$, was cleaved into two bands of approximately 55 and $30 \mathrm{kDa}$ (Figure $2 \mathrm{C})$. The heavier band was confirmed to be the L1-13 fragment by western blot, and the 30-kDa band was shown to be the Fc fragment (data not shown). In contrast to L1-16, some degradation of L1-13 was evident in SDS-PAGE gels and western blots of the cleavage reaction (Figure 2C and data not shown). It is possible that the L1-13 fragment may be intrinsically labile without the Fc, which is known to have a stabilizing influence on some proteins (2). For L113 , the ability to conduct cleavage reactions at $4{ }^{\circ} \mathrm{C}$ was highly advantageous, as this temperature appeared to improve its stability (data not shown).

To remove TEV protease from protein preparations, we took advantage of a poly-histidine tag that is present on the commercially available enzyme (8). A short incubation with metal affinity resin removed TEV protease from the cleav-
A

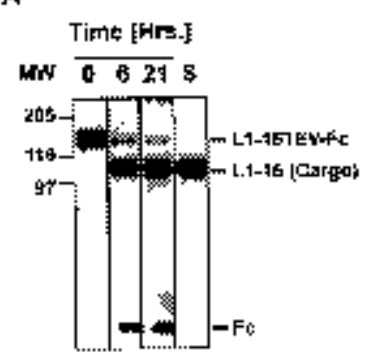

c

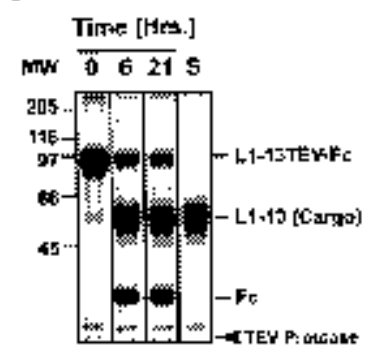

B



to

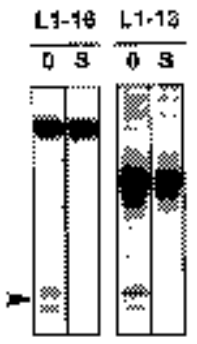

E

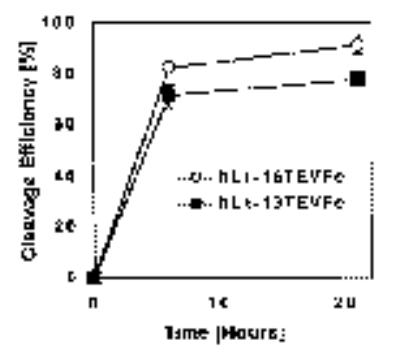

Figure 2. Cleavage of L1-TEV-Fc fusion proteins. (A) Analysis of L1-16TEV-Fc cleavage via SDSPAGE. Equal aliquots $(20 \mu \mathrm{L})$ of reaction slurry were analyzed after incubation with TEV protease for 0 , 6 , and $21 \mathrm{~h}$. An equivalent amount of supernatant (S), collected after $21 \mathrm{~h}$, was analyzed in parallel. (B) Western blots of L1-16TEV-Fc cleavage. Equal aliquots of the above samples were blotted with anti-L1 or anti-human $\mathrm{Fc}$ antibodies. In addition, equivalent fractions of proteins that remained bound to the protein-A beads after TEV cleavage (B) were analyzed in parallel. (C) SDS-PAGE gels of L1-13TEV-Fc cleavage. (D) Removal of TEV protease. Aliquots $(20 \mu \mathrm{L})$ of L1-16 and L1-13 preparations were analyzed before (0) and after (S) incubation with metal affinity resin. Note that TEV protease migrates as a doublet and is indicated by arrowheads. (E) Quantitation of cleavage efficiency. Values were obtained by densitometric analysis of gels, such as in (A) and (C), and data points represent mean cleavage efficiency averaged over $2-4$ independent reactions \pm SD. Note that mean efficiency (at $21 \mathrm{~h}$ ) for L1-16TEV-Fc was $90.6 \% \pm 4.1(n=4)$ and for L1-13TEV-Fc was $77.7 \% \pm 1.9(n=2)$. 
age reaction supernatants (Figure 2D). Therefore, highly pure L1 fragments were obtained by cleavage of TEV-Fc fusion proteins with TEV protease.

The neurite outgrowth activity of L1-16 and L1-13 was then assessed. For comparison, uncleaved L1-16TEVFc and L1-13TEV-Fc were tested in parallel. It was found that L1-16 strongly promoted neurite outgrowth, similar to L1-16TEV-Fc (Figure 3). Furthermore, L1-16 and L1-16TEV-Fc had similar potencies in this assay, as they were both active at concentrations as low as $22 \mathrm{nM}$ (data not shown). In contrast, neither L1-13 nor L1-13TEVFc promoted neurite outgrowth at concentrations as high as $600 \mathrm{nM}$ (Figure 3 and data not shown). These results show that the inactivity of L1-13TEV$\mathrm{Fc}$ was not due to artifactual effects of the Fc region. Instead, it is likely that additional L1 domains are necessary for neurite outgrowth activity, as was previously theorized (4).

The TEV-Fc fusion protein system yields highly pure monomeric proteins that are soluble in PBS. For many biological applications, these proteins are ready-to-use without further isolation or dialysis. The process is efficient as well as scalable: we have obtained yields as high as $2 \mathrm{mg} / \mathrm{L}$ cultured supernatant, with $90 \%$ cleavage efficiency, from individual L1-16 preparations (Figure 3E and data not shown). This is significant because certain protein-intensive tasks, such as X-ray crystallography or animal studies, require large quantities of homogenous proteins. Therefore, several useful applications of the TEV-Fc fusion protein system can be envisioned.

It should be noted that other cleavable Fc fusion proteins have been described, in which the $\mathrm{Fc}$ region can be removed by thrombin $(5,6)$ or subtilisin B PN' (1). However, the TEV-Fc system has several advantages, owing to the unique properties of TEV protease. First, the TEV protease cleavage site is longer (seven residues) and, hence, likely to be more specific than that of thrombin (four residues) or subtilisin (six residues). Second, cleavage of TEV-Fc fusion proteins can be conducted at $4^{\circ} \mathrm{C}$ (as opposed to $25^{\circ} \mathrm{C}$ or $37^{\circ} \mathrm{C}$ ), a temperature that preserves the stability of many proteins. Finally, commercially available TEV protease can be easily removed from protein preparations via its poly-histidine tag

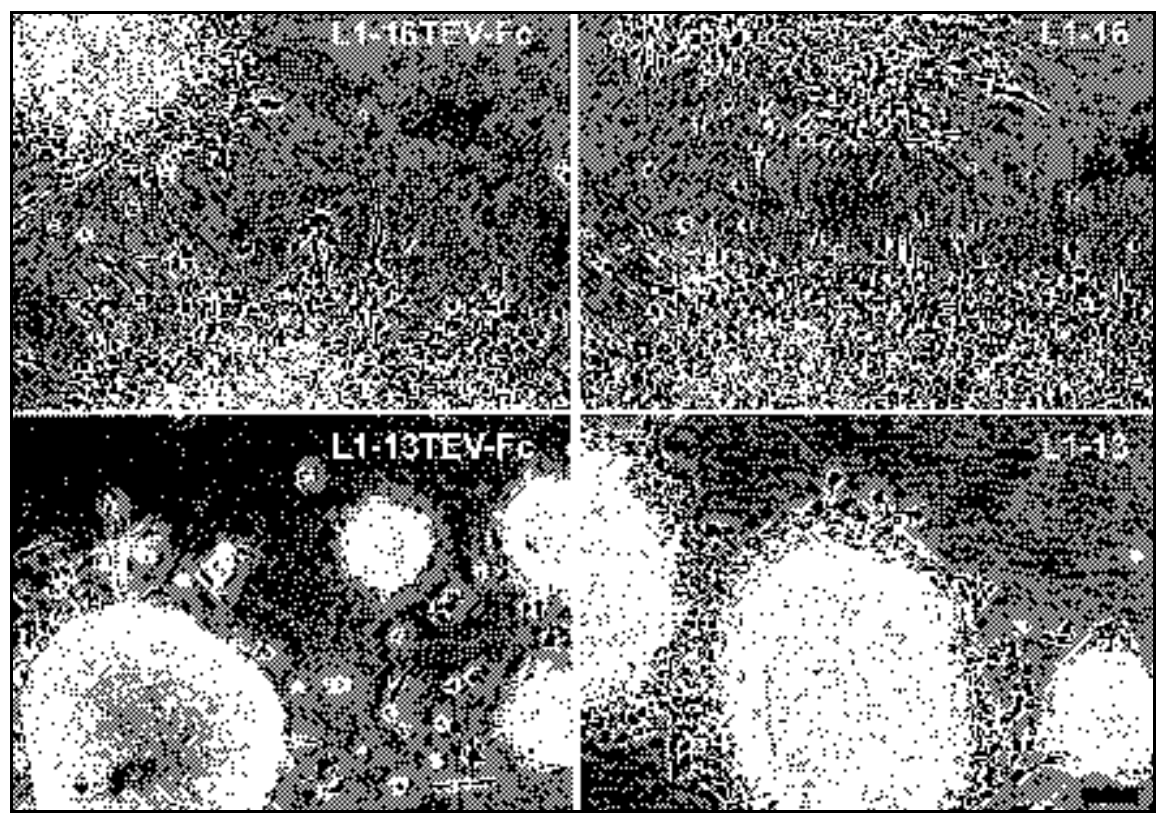

Figure 3. Neurite outgrowth activity of L1-TEV-Fc proteins and cleavage products. Representative pictures of mouse cerebellar reaggregates cultured on $200 \mathrm{nM}$ L1-TEV-Fcs or L1 fragments. The mean length of the tenth longest neurite (averaged over two experiments) was $139.7 \mu \mathrm{m} \pm 32.6$ (s E M) for L116TEV-Fc, $161.6 \pm 14.9$ for L1-16, and $0.0 \pm 0.0$ for L1-13TEV-Fc and L1-13. These later two treatments were indistinguishable from substrates coated with $1 \%$ BSA (which served as a negative control). $\operatorname{Bar}=75 \mu \mathrm{m}$

(8). Therefore, the TEV-Fc fusion protein system may be preferable to other cleavable Fc fusion proteins in certain situations. It is likely that TEV-Fc fusion proteins will further enhance the utility of this class of molecules for scientific and clinical applications.

\section{REFERENCES}

1.Beck, J.T., S.A. Marsters, R.J. Harris, P. Carter, A. Ashkenazi, and S.M. Chamow. 1994. Generation of soluble interleukin-1 receptor from an immunoadhesin by specific cleavage. Mol. Immunol. 31:1335-1344.

2.Chamow, S.M. and A. Ashkenazi. 1996. Im munoadhesins: principles and applications. Trends Biotechnol. 14:52-60.

3.Dwyer, M.A., A.J. Huang, C.Q. Pan, and R.A. Lazarus. 1999. Expression and characterization of a DNase I-Fc fusion enzyme. J. Biol. Chem. 274:9738-9743.

4.Haspel, J., D.R. Friedlander, N. Ivgy-May, S. Chickramane, C. Roonprapunt, S. Chen, M. Schachner, and M. Grumet. 2000. Critical and optimal Ig domains for promotion of neurite outgrowth by L1/Ng-CAM. J. Neurobiol. 42:287-302.

5.Hollenbaugh, D., J. Douthwright, V. McDonald, and A. Aruffo. 1995. Cleavable CD40Ig fusion proteins and the binding to sgp39. J. Immunol. Methods 188:1-7.

6.Jostock, T., G. Blinn, C. Renné, K.J. Kallen, S. Rose-John, and J. Mullberg. 1999. Im munoadhesins of interleukin- 6 and the IL6/soluble IL-6R fusion protein hyper-IL-6. J. Immunol. Methods 223:171-183.

7.Melcher, K. 2000. A modular set of prokaryotic and eukaryotic expression vectors. Anal. Biochem. 277:109-120.

8.Parks, T.D., K.K. Leuther, E.D. Howard, S.A. Johnston, and W.G. Dougherty. 1994 Release of proteins and peptides from fusion proteins using a recombinant plant virus protease. Anal. Biochem. 216:413-417.

9.Polayes, D., A. Goldstein, G. Ward, and A. Hughes. 1994. TEV protease, recombinant: a site-specific protease for efficient cleavage of affinity tags from expressed proteins. Focus 16:2-5.

10.Polayes, D., R. Harris, D. Anderson, and V. Ciccarone. 1996. New baculovirus expression vectors for the purification of recombinant protein from insect cells. Focus 18:10-13.

11.Sakurai, T., C. Roonprapunt, and M. Grumet. 1998. Purification of Ig-fusion proteins from medium containing Ig. BioTechniques 25:382-384.

12. Williams, E.J., P. Doherty, G. Turner, R.A. Reid, J.J. Hemperly, and F.S. Walsh. 1992. Calcium influx into neurons can solely account for cell contact-dependent neurite outgrowth stimulated by transfected L1. J. Cell Biol. 119:883-892.

We thank Dr. John Hemperly for providing Neuro 4.1 antibody and Dr. Melitta Schachner for providing human L1 cDNA. 


\section{Short Technical Reports}

We also thank Dr. Seema Dalal, Dr. Bruce Babiarz, and Joanne Babiarz for providing reagents and technical assistance. This work was funded by a grant from the Spinal Cord Research Foundation of the Paralyzed Veterans Association. Address correspondence to Dr. Martin Grumet, W.M. Keck Center for Collaborative Neuroscience, Rutgers, State University of New Jersey, 604 Allison Rd., Piscataway, NJ 08854-8082, USA.e-mail: mgrumet@rci.rutgers.edu

Received 5 July 2000; accepted 30 August 2000.

\author{
Jeffrey Haspel ${ }^{1,2}$, Cy Blanco 2 , \\ Jeffrey Jacob ${ }^{1}$, and \\ Martin Grumet ${ }^{1,2}$ \\ ${ }^{1}$ Rutgers State University \\ of New Jersey \\ Piscataway, NJ \\ ${ }^{2} N Y U$ Medical Center \\ New York, NY, USA
}

\section{Promoter Methylation Analysis on Micro- dissected Paraffin- Embedded Tissues Using Bisulfite Treatment and PCR-SSCP}

BioTechniques 30:66-72 (January 2001)

\begin{abstract}
Methylation-sensitive single-strand conformation analysis (MS-SSCA) is a new method of screening for DNA methylation changes. The combination of bisulfite modification and PCR results in the conversion of unmethylated cytosines to thymines, whereas methylated cytosines remain unchanged. This sequence conversion can
\end{abstract}

lead to methylation-dependent alterations of single-strand conformation, which can be detected by SSCA. An analysis of mixtures of methylated and unmethylated DNA at known ratios revealed that the relative intensities of the corresponding bands following MS-SSCA were maintained. MS-SSCA was applied for methylation analysis of human p16 promoter region using genomic DNA obtained from either frozen, fixed, or microdissected fixed tissue sections. MSSSCA is a rapid, specific, and semiquantitative approach that allows the detection of methylation of the pl6 gene promoter. In reconstruction experiments, the method permits the detection of $10 \%$ or less of cells harboring a methylated p16 promoter. We have been successful in analyzing by $M S$ SSCA almost all (96\%) tumor samples microdissected from archival paraffin-embedded fixed tissue sections and obtaining reproducible results. In addition, when microdissection was performed, the clonality of this genetic alteration could be identified. 